\title{
A Refined Geometry of Logic
}

\author{
DAVID MiLler \\ University of Warwick
}

\begin{abstract}
In order to measure the degree of dissimilarity between elements of a Boolean algebra, the author's (1984) proposed to use pseudometrics satisfying generalizations of the usual axioms for identity. The proposal is extended, as far as is feasible, from Boolean algebras (algebras of propositions) to Brouwerian algebras (algebras of deductive theories). The relation between Boolean and Brouwerian geometries of logic turns out to resemble in a curious way the relation between Euclidean and non-Euclidean geometries of physical space. The paper ends with a brief consideration of the problem of the metrization of the algebra of theories.
\end{abstract}

Keywords: Boolean algebra, Brouwerian algebra, metric, measure, partial identity.

\section{Background}

In (1984) I proposed an axiom system $\mathbb{A}$ for a function $\mathfrak{d}$ on a poset $\mathfrak{L}$ that in a rather straightforward manner generalizes and strengthens the usual elementary axioms for identity. The value of $\mathfrak{d}(a, c)$ can be understood as one way, by no means the only one, of grading the degree of dissimilarity or diversity of the elements $a$ and $c$ of $\mathfrak{L}$. The function $\mathfrak{d}$ is not required to be real-valued, but that is its most natural interpretation, in which case, as is easily demonstrated, it satisfies the usual requirements of a pseudometric operation. The system $\mathbb{A}$ was applied to an arbitrary Boolean algebra $\mathfrak{L}$, for which it was proved to be equivalent, given some appropriate definitions, to two other axiomatic systems: $\mathbb{B}$, a different and more familiar set of axioms for a pseudometric operation $\mathfrak{d}$; and $\mathbb{C}$, the standard system of axioms for an unnormalized measure or positive valuation function $\mu$. As was remarked, the close relation between the systems $\mathbb{B}$ and $\mathbb{C}$ in Boolean algebras (in which every strictly positive unnormalized measure is also a positive isotone valuation) is well known. The paper also drew attention to a number of variations and generalizations, some of them first mentioned in Miller (1979). Several of the derivations, for example, but not all of them, can be carried out when $\mathfrak{L}$ is assumed not to be a Boolean algebra, but only a lattice. It was noted that if $\mathfrak{L}$ is a lattice, the system $\mathbb{B}$, together with the assumption that the pseudometric $\mathfrak{d}$ is a metric (that is, $\mathfrak{d}(a, c)=0$ only if $a=c$ ), compels $\mathfrak{L}$ to be modular (Birkhoff 1967, Theorem X.2). It was observed also that if one of the axioms in $\mathbb{B}$ is strengthened from an inequality to an identity, then $\mathfrak{L}$

Principia 13(3): 339-56 (2009).

(C) Copyright D. W. Miller 2009 Published by NEL — Epistemology and Logic Research Group, Federal University of Santa Catarina (UFSC), Brazil. 
is forced to be distributive. The derivations, furthermore, were shown to be valid when the range of $\mathfrak{d}$ is not the real line but an arbitrary partially ordered abelian semigroup with unit and some weak cancellation properties.

The Boolean operation $\triangle$ of symmetric difference plays an important role in many of the derivations in the systems $\mathbb{A}$ and $\mathbb{B}$, and in the definition in the system $\mathbb{C}$ of the pseudometric $\mathfrak{d}$ in terms of the measure $\mu$. Yet no thought was given in (1984) to whether similar results could be obtained for Brouwerian (dual-intuitionistic) algebras, where too the symmetric difference operation is defined and is known to possess pseudometric properties (Nordhaus \& Lapidus 1954, Miller 1978, § 3). This was a conspicuous omission. The algebra of deductive theories of a language incorporating classical logic, whose Lindenbaum-Tarski algebra is Boolean, is known to be Brouwerian in general (provided that, as in the Lindenbaum-Tarski algebra, $\mathbf{X} \preceq \mathbf{Z}$ means that $\mathbf{X}$ is logically stronger than $\mathbf{Z}$ and not, as in the original presentation in Tarski 1935/1936, that $\mathbf{X} \subseteq \mathbf{Z}$ ). Since the problem that prompted the original investigation was to say something enlightening about the idea of the distance of a theory from the truth, or its distance from another theory (Miller 1977, 1978), it is plainly of interest to develop further the Brouwerian case.

Another matter that needs to be addressed is the question of the existence of a metric $\mathfrak{d}$ and of a strictly positive measure $\mu$. Pseudometrics satisfying the systems $\mathbb{A}$ and $\mathbb{B}$ abound, as do measures satisfying the system $\mathbb{C}$. We certainly need to be more specific. But as recorded in Miller (1986), p.174, these two goals are not easily attainable together. Unless the Brouwerian algebra $\mathfrak{L}$ is Boolean, there exists no metric $\mathfrak{d}$ obeying the axiom system $\mathbb{A}$, even if $\mathfrak{L}$ is finite.

In this paper the axiom systems $\mathbb{A}$ and $\mathbb{C}$ will be replaced by systems $\mathbb{A}^{\star}$ and $\mathbb{C}^{\star}$ that overcome the main difficulties sketched. $\mathbb{A}^{\star}$ and $\mathbb{C}^{\star}$ will be shown to be logically equivalent in Boolean algebras to $\mathbb{A}$ and $\mathbb{C}$ respectively (and therefore to $\mathbb{B}$ ), and to be logically equivalent in Brouwerian algebras to each other. The system $\mathbb{B}$ will be shown to be equivalent in Boolean algebras to a somewhat more transparent system $\mathbb{B}^{\natural}$, which in its turn can be modified to a system $\mathbb{B}^{\natural \star}$ that is appropriate to Brouwerian algebras. We shall note that there exists another way of providing axioms for pseudometric operations in Brouwerian algebras, which abandons the system $\mathbb{A}$ and retains the system $\mathbb{B}$ without modification (and makes a trifling change to the system $\mathbb{C}$ here presented). Results obtained by Mormann (2006) under this alternative approach will be adapted to show that every Brouwerian algebra of theories (of a denumerable language) can be metrized by a metric that complies with the characteristic axiom $\left(\mathbb{A}^{\star} 1\right)$ of the system $\mathbb{A}^{\star}$.

Principia 13(3): 339-56 (2009). 


\section{Three Axiom Systems}

The letters $a, b, c$ are used as variables for the elements of an arbitrary lattice $\mathfrak{L}$ with ordering $\preceq$. The join and meet operations of $\mathfrak{L}$ are represented by + and . respectively, and the unit and zero elements (if these elements exist) by $\top$ and $\perp$ respectively. The symbols + and $=$ are used also for operations in some partially ordered semigroup with unit, the non-negative real numbers unless otherwise stated, which is the space of values for two functions $\mathfrak{d}$ and $\mu$ defined on $\mathfrak{L}$. There should not be any danger of confusion between the different uses of these symbols.

We first present the three axiom systems $\mathbb{A}, \mathbb{B}, \mathbb{C}$ already mentioned. The axioms of $\mathbb{A}$ are:

$$
\begin{aligned}
\mu(b) & ={ }_{\text {Df }} \mathfrak{d}(b, \perp) \\
\mathfrak{d}(a, c)+\mathfrak{d}(\varphi a, b) & \geq \mathfrak{d}(\varphi c, b) \\
a \succeq b \succeq c & \Rightarrow \mathfrak{d}(a, c)=\mathfrak{d}(a, b)+\mathfrak{d}(b, c),
\end{aligned}
$$

where in $(\mathbb{A} 1)$, which is a scheme of axioms, the term $\varphi c$ is identical with the term $\varphi a$ except perhaps for containing $c$ at one or more places at which $\varphi$ a contains $a$. The axioms of $\mathbb{B}$ are:

$$
\begin{aligned}
\mu(b) & ={ }_{\text {Df }} \mathfrak{d}(b, \perp) \\
\mathfrak{d}(b, a)+\mathfrak{d}(b, c) & \geq \mathfrak{d}(a, c) \\
\mathfrak{d}(a, c) & \geq \mathfrak{d}(b+a, b+c)+\mathfrak{d}(b \cdot a, b \cdot c) .
\end{aligned}
$$

This system of axioms ( $\mathbb{B} 1$ is just the triangle inequality) derives from Theorem 1 of Chapter X of Birkhoff (1967). Note that the definition ( $\mathbb{B} 0$ ) of $\mu$ in terms of $\mathfrak{d}$ is identical with $(\mathbb{A} 0)$. $\mathbb{C}$ is a familiar system of axioms for a measure $\mu$. The term $a \Delta c$ in the definition ( $\mathbb{C} 0$ ) of $\mathfrak{d}$ in terms of $\mu$ is the Boolean symmetric difference (exclusive disjunction) $a \cdot c^{\prime}+c \cdot a^{\prime}$ of $a$ and $c$.

$$
\begin{aligned}
\mathfrak{d}(a, c) & { }_{\text {Df }} \mu(a \Delta c) \\
\mu(\perp) & =0 \\
\mu(b) & \geq 0 \\
\mu(a)+\mu(c) & =\mu(a+c)+\mu(a \cdot c) .
\end{aligned}
$$

If the lattice $\mathfrak{L}$ is a Boolean algebra, the systems $\mathbb{A}, \mathbb{B}$, and $\mathbb{C}$ are logically equivalent (Miller 1984). In $\S 3$ we shall add a fourth equivalent system $\mathbb{B}^{\natural}$, like $\mathbb{B}$ but somewhat more transparent. 


\section{Degrees of Diversity}

The axiom systems $\mathbb{B}$ and $\mathbb{C}$ need no immediate discussion, but something must be said about $\mathbb{A}$. In elementary logic the relation of identity or equality is standardly introduced by the axioms

$$
\begin{aligned}
b & =b \\
a=c \wedge \Phi a & \Rightarrow \Phi c,
\end{aligned}
$$

where $\Phi a$ is a formula perhaps involving $a$, and $\Phi c$ is like $\Phi a$ except perhaps for containing $c$ free at one or more places where $\Phi a$ contains $a$ free. If we write $\mathbb{O}$ for the falsum or absurdity (of the metalanguage), and $\Leftarrow$ for the converse of $\Rightarrow(\Leftarrow$ is to be read if and $\Rightarrow$ is to be read if ... then), then we may accordingly characterize also the relation $\neq$ of diversity by the axioms

$$
\begin{aligned}
b \neq b & \Leftrightarrow \mathbb{O} \\
a \neq c \vee \neg \Phi a & \Leftarrow \neg \Phi c .
\end{aligned}
$$

In the case of algebraic languages, in which all atomic formulas are identities, the second principle $(\mathbb{E} 3)$, the principle of the diversity of discernibles, may be rendered in one of the forms

$$
\begin{aligned}
a \neq c \vee \varphi a \neq \psi a & \Leftarrow \varphi c \neq \psi c \\
a \neq c \vee \varphi a \neq b & \Leftarrow \varphi c \neq b,
\end{aligned}
$$

where $\varphi a$ and $\psi a$ are terms perhaps involving $a$ and the conventions on substitution are as before. In general these statements are not logically equivalent; ( $\mathbb{E} 5$ ) is weaker than $(\mathbb{E} 4)$. A theory of the partial diversity or degree of dissimilarity of two elements of an algebra may be obtained by generalizing these axioms $(\mathbb{E} 0)$ and $(\mathbb{E} 4)$ in terms of a (typically real-valued) function $\mathfrak{d}$ satisfying

$$
\begin{aligned}
\mathfrak{d}(b, b) & =0 \\
\mathfrak{d}(a, c)+\mathfrak{d}(\varphi a, \psi a) & \geq \mathfrak{d}(\varphi c, \psi c) .
\end{aligned}
$$

In Boolean algebras, the scheme $(\mathbb{E} 7)$ is equivalent to the restricted version called (A1) above:

$$
\mathfrak{d}(a, c)+\mathfrak{d}(\varphi a, b) \geq \mathfrak{d}(\varphi c, b) .
$$

When the axiom $(\mathbb{E} 6)$ and the scheme $(\mathbb{E} 7)$ are augmented by the obvious definition of identity,

$$
a=c={ }_{\mathrm{Df}} \mathfrak{d}(a, c)=0,
$$

Principia 13(3): 339-56 (2009). 
they yield a set of axioms for degrees of dissimilarity or diversity. In the bulk of this paper the westward half of $(\mathbb{E} 9)$ is not assumed, and it is only in $\S 6$ that metrics come to the fore.

Consider first a degenerate algebra, with no operations. As $a$ is the only term that involves $a$,

$$
\begin{aligned}
\mathfrak{d}(a, c)+\mathfrak{d}(a, a) & \geq \mathfrak{d}(c, a), \\
\mathfrak{d}(a, c) & =\mathfrak{d}(c, a), \\
\mathfrak{d}(a, c)+\mathfrak{d}(a, b) & \geq \mathfrak{d}(c, b),
\end{aligned}
$$

are all we can extract from $(\mathbb{E} 6)$ and $(\mathbb{E} 7)$. In other words, $\mathfrak{d}$ is a pseudometric operation. If ( $\mathbb{E} 9)$ holds too, then $\mathfrak{d}$ is a metric operation. If $\mathfrak{d}$ is defined on an algebra $\mathfrak{L}$ that contains operations, a strong condition of uniform continuity $(\mathbb{E} 14)$, the Lipschitz condition, is satisfied:

$$
\begin{aligned}
\mathfrak{d}(a, c)+\mathfrak{d}(\varphi a, \varphi a) & \geq \mathfrak{d}(\varphi c, \varphi a) \\
\mathfrak{d}(a, c) & \geq \mathfrak{d}(\varphi a, \varphi c) \\
\mathfrak{d}(a, c) & \geq \mathfrak{d}(b, b)=0
\end{aligned}
$$

$\mathbb{E} 6, \mathbb{E} 14$

Inequality $(\mathbb{E} 14)$, although weaker than $(\mathbb{E} 7)$, is easier to work with than either $(\mathbb{E} 7)$ or $(\mathbb{E} 8)$. It follows from $(\mathbb{E} 8)$ alone, and not until step $(\mathbb{A} 5)$ below is the full strength of $(\mathbb{E} 7)$ needed. Note also that from $(\mathbb{E} 14)$ and the triangle inequality $(\mathbb{E} 12)$ we can derive $(\mathbb{E} 8)$, but not $(\mathbb{E} 7)$.

The next results invoke the assumption that the algebra $\mathfrak{L}$ is a lattice with join + and meet $\cdot$.

$$
\begin{array}{rlr}
\mathfrak{d}(a, c) & \geq \mathfrak{d}(b+a, b+c) & \mathbb{E} 14 \\
\mathfrak{d}(a, c) & \geq \mathfrak{d}(a \cdot b, c \cdot b) & \mathbb{E} 14 \\
\mathfrak{d}(c, a \cdot c) & \geq \mathfrak{d}(a+c, a+a \cdot c) & \mathbb{E} 16 \\
\mathfrak{d}(c, a \cdot c) & \geq \mathfrak{d}(a+c, a) & \mathbb{E} 18 \\
\mathfrak{d}(a+c, a) & \geq \mathfrak{d}((a+c) \cdot c, a \cdot c) & \mathbb{E} 17 \\
\mathfrak{d}(a+c, a) & \geq \mathfrak{d}(c, a \cdot c) & \mathbb{E} 20 \\
\mathfrak{d}(a+c, a) & =\mathfrak{d}(c, a \cdot c) & \mathbb{E} 19, \mathbb{E} 21
\end{array}
$$

The lattice quadrangles are parallelograms. The isotony principles $(\mathbb{E} 23)$ and $(\mathbb{E} 24)$ follow easily.

$$
\begin{aligned}
& a \succeq b \succeq c \Rightarrow \mathfrak{d}(a, c) \geq \mathfrak{d}(a, b) \\
& a \succeq b \succeq c \Rightarrow \mathfrak{d}(a, c) \geq \mathfrak{d}(b, c) .
\end{aligned}
$$

Principia 13(3): 339-56 (2009). 


\section{Radial Convexity}

The metric part of the axiomatic system $\mathbb{A}$ presented in $\S 1$ above is obtained by adding to the scheme $(\mathbb{A} 1)$, which was labelled $(\mathbb{E} 8)$ in $\S 2$, the following principle of additivity along chains:

$$
a \succeq b \succeq c \Rightarrow \mathfrak{d}(a, c)=\mathfrak{d}(a, b)+\mathfrak{d}(b, c) .
$$

Since all the metrical formulas $(\mathbb{E} 6)-(\mathbb{E} 24)$ of $\S 2$ hold for the trivial metric (whose value is 0 when $a=c$ and 1 when $a \neq c)$, ( $\mathbb{A} 2)$ is an independent assumption. Mormann (2006), definition 2.6, calls those (pseudo)metric operations $\mathfrak{d}$ for which (A2) holds radially convex (pseudo)metrics.

In Miller (1984) there is a short proof that, if $\mathfrak{L}$ is a Boolean algebra, the scheme $(\mathbb{A} 1)$ and the axiom $(\mathbb{A} 2)$ imply that the lattice quadrilaterals are rectangles (see $\S 1$, lines $14-21)$. It is shown also that this result, which is proved in formula $(\mathbb{A} 8)$ below, is not forthcoming for arbitrary lattices, even Boolean lattices. Yet the result is demonstrable for all lattices once the scheme $(\mathbb{A} 1)$, formerly called $(\mathbb{E} 8)$, is strengthened to $(\mathbb{E} 7)$, which we here formally relabel:

$$
\mathfrak{d}(a, c)+\mathfrak{d}(\varphi a, \psi a) \geq \mathfrak{d}(\varphi c, \psi c)
$$

Since these two schemes $(\mathbb{A} 1)$ and $\left(\mathbb{A}^{\star} 1\right)$ are logically equivalent in Boolean algebras (Miller op.cit., formula 23 ), and it is $\left(\mathbb{A}^{\star} 1\right)$ that we shall eventually want to adopt for other algebras, we shall work in this section in the axiomatic system $\mathbb{A}^{+}$whose metrical postulates are $\left(\mathbb{A}^{\star} 1\right)$ and $(\mathbb{A} 2)$ (the definitional axiom $(\mathbb{A} 0)$ will not be needed). Our purpose is to show how Birkhoff's complicated axiom ( $\mathbb{B} 2$ ) may be simplified, whatever the lattice; in the presence of $(\mathbb{B} 1)$, it obviously implies radial convexity $(\mathbb{A} 2)$, but it is itself a consequence of $(\mathbb{A} 2)$ and rectangularity.

$(\mathbb{E} 22)$ states that the opposite sides of a typical lattice quadrilateral are of equal length. We show now, within the system $\mathbb{A}^{+}$, that the diagonals of the quadrilateral also are of equal length.

$$
\begin{aligned}
& \mathfrak{d}(b, b)+\mathfrak{d}(b, b)=\mathfrak{d}(b, b) \\
& \mathfrak{d}(b, b)=0 \\
& \text { A3 } \\
& \mathfrak{d}(a, c)+\mathfrak{d}(a+a, a \cdot a) \geq \mathfrak{d}(a+c, a \cdot c) \quad \mathbb{A}^{\star} 1 \\
& \mathfrak{d}(a+c, a \cdot c)+\mathfrak{d}(a+c+a, a+c+c) \geq \mathfrak{d}(a \cdot c+a, a \cdot c+c) \quad \mathbb{A}^{\star} 1 \\
& \mathfrak{d}(a+c, a \cdot c) \geq \mathfrak{d}(a, c) \\
& \mathfrak{d}(a, c)=\mathfrak{d}(a+c, a \cdot c) \text {. } \\
& \mathbb{A} 4, \mathbb{A} 5, \mathbb{A} 7
\end{aligned}
$$

Principia 13(3): 339-56 (2009). 


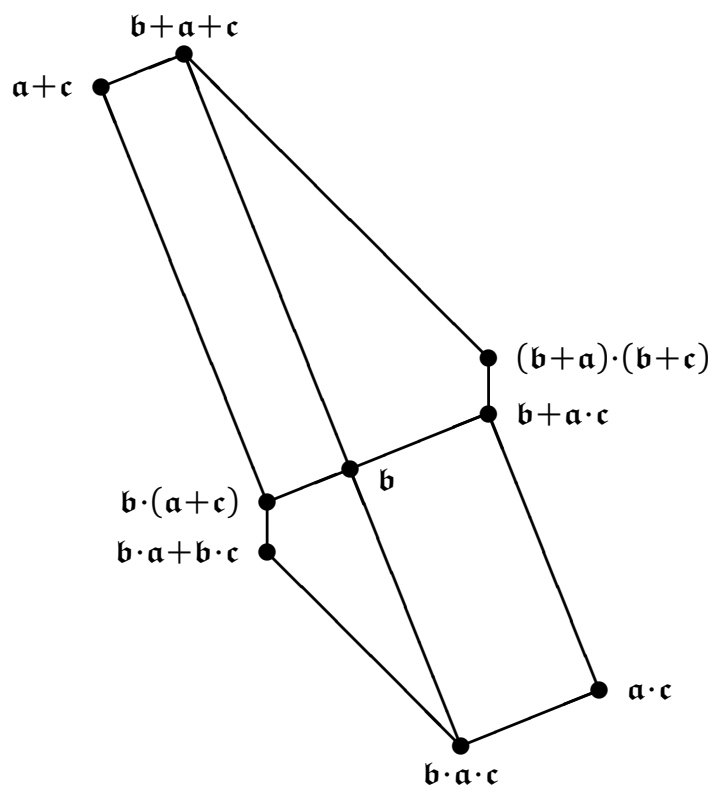

Figure 0: A diagrammatic demonstration that $(\mathbb{B} 2)$ follows from radial convexity plus the rectangularity of the lattice quadrilaterals.

We are now able to give a proof of Birkhoff's axiom $(\mathbb{B} 2)$ within the system $\mathbb{A}^{+}$, and further to show that, if the lattice $\mathfrak{L}$ is distributive, then the inequality in $(\mathbb{B} 2)$ is turned into an identity:

$$
\mathfrak{d}(a, c)=\mathfrak{d}(b+a, b+c)+\mathfrak{d}(b \cdot a, b \cdot c) .
$$

By (A8), $\mathfrak{d}(a, c)=\mathfrak{d}(a+c, a \cdot c)$. Figure 0 shows that if to the two ends of the interval $[a+c, a \cdot c]$ the intervals $[b+a+c, a+c]$ and $[a \cdot c, b \cdot a \cdot c]$ are added, we obtain a path through the lattice with length, by radial convexity $(\mathbb{A} 2)$, equal to

$$
\begin{gathered}
\mathfrak{d}(b+a+c,(b+a) \cdot(b+c))+\mathfrak{d}((b+a) \cdot(b+c), b+a \cdot c)+ \\
+\mathfrak{d}(b+a \cdot c, b)+\mathfrak{d}(b, b \cdot(a+c))+ \\
+\mathfrak{d}(b \cdot(a+c), b \cdot a+b \cdot c)+\mathfrak{d}(b \cdot a+b \cdot c, b \cdot a \cdot c) .
\end{gathered}
$$

The two middle terms here are, by $(\mathbb{E} 22)$, equal in length to the two added intervals, while the two outer terms are, by (A8), equal in length to $\mathfrak{d}(b+a, b+c)$ and $\mathfrak{d}(b \cdot a, b \cdot c)$. The other two terms are non-negative, and if $\mathfrak{L}$ is distributive, zero.

Birkhoff's axiom $(\mathbb{B} 2)$ is therefore derivable from the formulas $(\mathbb{A} 2),(\mathbb{E} 22)$, and $(\mathbb{8} 8)$. It is clear that $(\mathbb{B} 2)$ is satisfied by any function $\mathfrak{d}$ that has a constant negative value (say, -1 ), and hence it cannot ensure the triangular inequality, which above 
was called $(\mathbb{B} 1)$, or even $\mathfrak{d}(b, b)=0$, which was called $(\mathbb{E} 6)$. Since $(\mathbb{E} 6)$ follows from $(\mathbb{A} 2)$, we have to assume more than $(\mathbb{B} 2)$ if we are to prove $(\mathbb{A} 2)$. We shall show that the three formulas $(\mathbb{A} 2),(\mathbb{E} 22)$, and $(\mathbb{A} 8)$ are all derivable from $(\mathbb{B} 2)$ together with $(\mathbb{B} 1)$; that is, that they are theorems of the system $\mathbb{B}$. The following lines correct an error in the derivation on lines $0-15$ and 26-31 of $\S 2$ of Miller (1984).

$$
\begin{array}{rlr}
\mathfrak{d}(b, a)+\mathfrak{d}(b, c) & \geq \mathfrak{d}(a, c) & \\
\mathfrak{d}(a, c) & \geq \mathfrak{d}(b+a, b+c)+\mathfrak{d}(b \cdot a, b \cdot c) & \mathbb{B} 2 \\
a \succeq b \succeq c & \Rightarrow \mathfrak{d}(a, c) \geq \mathfrak{d}(a, b)+\mathfrak{d}(b, c) & \mathbb{B} 1, \mathbb{B} 2 \\
\mathfrak{d}(b, b)+\mathfrak{d}(b, b) & =\mathfrak{d}(b, b) & \mathbb{B} 4 \\
\mathfrak{d}(b, b) & =0 & \mathbb{B} 1, \mathbb{B} 5 \\
\mathfrak{d}(b, a) & =\mathfrak{d}(a, b) & \mathbb{B} 1, \mathbb{B} 3, \mathbb{B} 6 \\
a \succeq b \succeq c & \Rightarrow \mathfrak{d}(a, c)=\mathfrak{d}(a, b)+\mathfrak{d}(b, c) & \mathbb{B} 1, \mathbb{B} 5 \\
\mathfrak{d}(b, a) & \geq 0 & \mathbb{B} 2, \mathbb{B} 8 \\
\mathfrak{d}(a, c) & \geq \mathfrak{d}(b \cdot a, b \cdot c) & \mathbb{B} 9 \\
\mathfrak{d}(a+c, a) & \geq \mathfrak{d}(c \cdot(a+c), c \cdot a) & \mathbb{B} 2, \mathbb{B} 8 \\
\mathfrak{d}(a, c) & \geq \mathfrak{d}(b+a, b+c) & \mathbb{B} 11 \\
\mathfrak{d}(c, a \cdot c) & \geq \mathfrak{d}(a+c, a+a \cdot c) & \mathbb{B} 10, \mathbb{B} 12 \\
\mathfrak{d}(a+c, a) & =\mathfrak{d}(c, a \cdot c) & \mathbb{B} 2 \\
\mathfrak{d}(a, c) & \geq \mathfrak{d}(a+a, a+c)+\mathfrak{d}(a \cdot a, a \cdot c) & \mathbb{B} 1, \mathbb{B} 14 \\
\mathfrak{d}(a, c) & \geq \mathfrak{d}(a+c, a \cdot c) & \mathbb{B} 2 \\
\mathfrak{d}(a+c, a \cdot c) & \geq \mathfrak{d}((a \cdot c)+a,(a \cdot c)+c)+ & \mathbb{B} 5, \mathbb{B} 15, \mathbb{B} 16 \\
\mathfrak{d}(a, c) & =\mathfrak{d}(a+c, a \cdot c) &
\end{array}
$$

The formulas to be proved, $(\mathbb{A} 2),(\mathbb{E} 22)$, and $(\mathbb{A} 8)$, are at lines $(\mathbb{B} 7),(\mathbb{B} 13)$, and $(\mathbb{B} 17)$, respectively.

These results encourage the recognition of a fourth axiom system $\mathbb{B}^{\natural}$, a notable simplification of the system $\mathbb{B}$, and equivalent to each of $\mathbb{A}, \mathbb{B}$, and $\mathbb{C}$ in Boolean algebras. $\mathbb{B}^{\natural}$ has five axioms, the definition $\left(\mathbb{B}^{\natural} 0\right)$, and the principles of triangularity $\left(\mathbb{B}^{\natural} 1\right)$, translation invariance $\left(\mathbb{B}^{\natural} 2\right)$, torsion invariance $\left(\mathbb{B}^{\natural} 3\right)$, and radial convexity $\left(\mathbb{B}^{\natural} 4\right)$ :

$$
\begin{aligned}
\mu(b) & ={ }_{\mathrm{Df}} \mathfrak{d}(b, \perp) \\
\mathfrak{d}(b, a)+\mathfrak{d}(b, c) & \geq \mathfrak{d}(a, c) \\
\mathfrak{d}(a+c, a) & =\mathfrak{d}(c, a \cdot c)
\end{aligned}
$$

Principia 13(3): 339-56 (2009). 


$$
\begin{aligned}
\mathfrak{d}(a, c) & =\mathfrak{d}(a+c, a \cdot c) \\
a \succeq b \succeq c & \Rightarrow \mathfrak{d}(a, c)=\mathfrak{d}(a, b)+\mathfrak{d}(b, c) .
\end{aligned}
$$

\section{Brouwerian Algebras}

Although, as we have seen, a good number of the derivations succeed in all lattices, the investigation in Miller (1984) was carried out, for the most part, under the hypothesis that the lattice $\mathfrak{L}$ is a Boolean algebra. For the rest of the paper we shall loosen this hypothesis, and assume only that the lattice on which $\mathfrak{d}$ is defined is a Brouwerian algebra; that is to say, $\mathfrak{L}$ is a lattice with unit $T$ in which for every two elements $a, c$ there exists a smallest element $b$ such that $b+c \succeq a$. This element, written $a-c$, is called the remainder or difference when $c$ is subtracted from $a$. Brouwerian algebras are dual to Heyting algebras, the algebras corresponding to intuitionistic logic (in which for every two elements $a, c$ there is a largest element $b$, the conditional $a \rightarrow c$, such that $b \cdot a \preceq c$ ). Brouwerian algebras, like Heyting algebras, are invariably distributive, and all finite distributive lattices, in particular finite chains, are Brouwerian algebras. Just as in a Heyting algebra each element $b$ has a pseudocomplement $b \rightarrow \perp$ that obeys the law of non-contradiction $b \cdot(b \rightarrow \perp)=\perp$, so in a Brouwerian algebra each element $b$ has an authentic complement $T-b$ that obeys the law of excluded middle $b+(T-b)=T$; it will be called the authocomplement of $b$, and written $b^{\prime}$. The symmetric difference of $a$ and $c$, which is defined by

$$
a \Delta c={ }_{\mathrm{Df}}(a-c)+(c-a),
$$

will continue to play a crucial role in what we do. It must not be forgotten that in Brouwerian algebras neither of the Boolean identities $a-c=a \cdot c^{\prime}$ and $a \triangle c=$ $a \cdot c^{\prime}+c \cdot a^{\prime}$ is generally valid.

Because of the relative unfamiliarity of the laws of the non-Boolean remainder and symmetric difference $(\mathbb{D} 0$ ), we list below without proof the main ones appealed to in the rest of the paper. They are duals of perhaps more homely laws of the intuitionistic conditional and biconditional. Because of its importance, attention should be drawn to the law $(\mathbb{D} 10)$, in which $\chi c$ is a term like $\chi a$ except for containing $c$ at one or more places where $\chi a$ contains $a$. This is the dual of the intuitionistic law of replacement: $p \leftrightarrow r \vdash \mathrm{X} p \leftrightarrow \mathrm{X} r$, where $\mathrm{X} p$ is a formula that contains free the variable $p$ and $\mathrm{X} r$ is the result of replacing one or more instances of $p$ by the variable $r$.

$$
\begin{aligned}
& a-c=(a+c)-c \\
& a-c=a-a \cdot c
\end{aligned}
$$

Principia 13(3): 339-56 (2009). 


$$
\begin{aligned}
(a-c) \cdot c & =(a-c) \cdot(a \cdot c) \\
a \triangle c & =(a+c) \Delta(a \cdot c) \\
a+c & =(a \triangle c)+a \cdot c \\
a \triangle c & \geq(a \triangle b)+(b \triangle c) \\
a \succeq b \succeq c & \Rightarrow a \Delta c=(a \Delta b)+(b \Delta c) \\
a \succeq c & \Leftrightarrow(a \Delta c)+c=a \\
a \succeq c \Rightarrow a \triangle c & =a-c \\
a \Delta c & \succeq \chi a \Delta \chi c \\
\top^{\prime} & =\perp \\
\left(b \cdot b^{\prime}\right)^{\prime} & =\top \\
b-b & =\perp \\
b-\perp & =b \\
b \triangle \perp & =b \\
b \triangle b & =\perp
\end{aligned}
$$

In the deductively more exiguous setting of Brouwerian algebras the equivalence of $\mathbb{A}, \mathbb{B}$, and $\mathbb{C}$ breaks down at a number of points. The most significant difficulty is that what distinguishes Boolean from non-Boolean Brouwerian algebras is the presence in the latter of at least one element $b$ for which the law of non-contradiction $b \cdot b^{\prime}=\perp$ fails. But $(\mathbb{D} 12)\left(b \cdot b^{\prime}\right)^{\prime}=\top$ is universally valid. As $(\mathbb{E} 11)$ and $(\mathbb{E} 14)$ above show, it follows from $(\mathbb{A} 1)$ that $\mathfrak{d}(a, c) \geq \mathfrak{d}(\varphi c, \varphi a)$, and therefore by $(\mathbb{D} 11)$ that $\mathfrak{d}\left(T, b \cdot b^{\prime}\right)$ $\geq \mathfrak{d}\left(\left(b \cdot b^{\prime}\right)^{\prime}, T^{\prime}\right)=\mathfrak{d}(T, \perp)$. By $(\mathbb{A} 2)$ we may conclude that $\mathfrak{d}\left(b \cdot b^{\prime}, \perp\right)=0$. That is to say, on a proper Brouwerian algebra $\mathfrak{L}$, as we shall henceforth call a Brouwerian algebra that is not Boolean, there exists no genuine metric (as opposed to pseudometric) satisfying the axiom system $\mathbb{A}$. If conformity with $\mathbb{A}$ is required, then the Brouwerian algebra is effectively booleanized. Yet system $\mathbb{B}$ certainly admits a metric $\mathfrak{d}$, and system $\mathbb{C}$ admits a measure $\mu$ that is positive isotone (and therefore strictly positive). For the simplest of all proper Brouwerian algebras, the three-element chain, possible val-

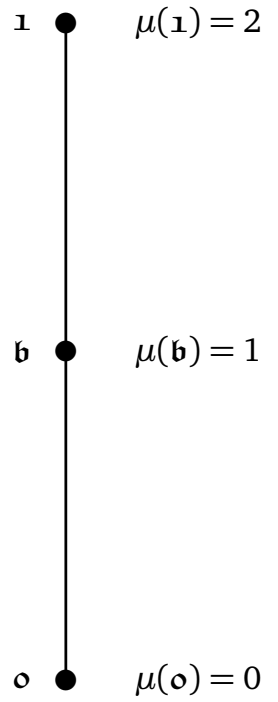

Figure 1: The threeelement chain ues of $\mu$ are recorded in Figure 1. For each $a \geq c$, we may set $\mathfrak{d}(a, c)=\mu(a)-\mu(c)$. In this algebra, with this metric, the authocomplement of $\mathfrak{b}$ is the unit element 1 , and so $\mathfrak{b} \cdot \mathfrak{b}^{\prime}=\mathfrak{b}$. The axioms of $\mathbb{A}$ are therefore transgressed. 
In a Brouwerian algebra, that is, neither $\mathbb{B}$ nor $\mathbb{C}$ implies $\mathbb{A}$. Nor does $\mathbb{A}$ imply either $\mathbb{B}$ or $\mathbb{C}$, though this can be taken care of by strengthening ( $\mathbb{A} 1$ ) to the already familiar generalization,

$$
\mathfrak{d}(a, c)+\mathfrak{d}(\varphi a, \psi a) \geq \mathfrak{d}(\varphi c, \psi c)
$$

It may be noted again that this alternative formulation makes no difference in Boolean algebras, where $\left(\mathbb{A} 1^{\star}\right)$ follows from $(\mathbb{A} 1$ ) (without the use of $\mathbb{A} 2$ ). Another shortcoming, which is relatively easily taken care of, is the failure of $\mathbb{C}$ to imply $\mathbb{B}$. To recover for Brouwerian algebra this implication we have to make two reforms; one is to replace the definition $(\mathbb{C} 0)$ with the variant

$$
\mathfrak{d}(a, c)=_{\mathrm{Df}} \mu(a+c)-\mu(a \cdot c),
$$

(Birkhoff op.cit., Theorem X.1), and the other is to add an axiom (to be called ( $\mathbb{C}^{\star} 4$ ) below) that states that the measure $\mu$ respects the ordering $\succeq$ (that is, $\mu$ is isotone). Neither of these changes can be thought of as momentous amendments to the original system $\mathbb{C}$, since in Boolean algebras $(\mathbb{C} 0)$ and $\left(\mathbb{C}^{+} 0\right)$ are logically equivalent given the other axioms (since $a \cdot c$ and $a \Delta c$ are disjoint), and $\left(\mathbb{C}^{\star} 4\right)$ is a consequence of these other axioms (since $a \cdot c$ and $a \cdot c^{\prime}$ are disjoint).

It is not difficult to see in what way the definition $\left(\mathbb{C}^{+} 0\right)$ differs from $(\mathbb{C} 0)$ in Brouwerian algebras. $(\mathbb{D} 5)(a \triangle c)+a \cdot c=a+c$ is a theorem of Brouwerian algebra. By additivity $(\mathbb{C} 3)$,

$$
\begin{aligned}
& \mu(a \Delta c)+\mu(a \cdot c)=\mu(a+c)+\mu((a \Delta c) \cdot(a \cdot c)) \\
& \mu(a+c)-\mu(a \cdot c)=\mu(a \Delta c)-\mu((a \Delta c) \cdot(a \cdot c))
\end{aligned}
$$

Since $(a \triangle c) \cdot(a \cdot c)=\perp$ is not a valid identity in Brouwerian algebra, in general $\left(\mathbb{C}^{+} 0\right)$ and $(\mathbb{C} 0)$ are distinct ways to define the function $\mathfrak{d}$. An explicit axiom of isotony, such as $\left(\mathbb{C}^{\star} 4\right)$, is needed in Brouwerian algebras because axiom $(\mathbb{C} 3)$ is vacuous in a chain. In the chain depicted in Figure 1 , for example, $\mu(\mathfrak{b})$ and $\mu(\mathbf{I})$ may be independently assigned any non-negative values.

It is apparent that the kinship in Boolean algebras between distance functions (and measures) and the more abstract degrees of dissimilarity characterized by the axiom system $\mathbb{A}$ cannot exist in Brouwerian algebras unless these axioms are somehow weakened. The natural way to proceed is to weaken axiom $(\mathbb{A} 1)$, even though, as we have just noted, it has to be strengthened as well. After all, the central problem arises from the discontinuity within Brouwerian algebras of the operations of authocomplementation and remainder (Miller 1986); from the fact, already visible in Figure 1, that adjacent elements (there 1 and $\mathfrak{b}$ ) can have authocomplements (there $\mathcal{O}$ and 1 ) that are not adjacent. This phenomenon seems to be blatantly in 
conflict with the intuitive idea behind $(\mathbb{A} 1)$, that if $a$ and $c$ are similar then there is an upper bound to how dissimilar $\varphi a$ and $\varphi c$ can be. None of this can be denied. Note however that the three-element chain is quite representative of all finite chains: if $\mathfrak{y}$ is the immediate inferior of the unit element 1 , then $\mathfrak{y}^{\prime}=1$ and $\mathfrak{l}^{\prime}=\mathcal{O}$ are as widely separated by the lattice ordering $\succeq$ as they can be. In other words, there is no upper bound in Brouwerian algebras to the number of elements (or equivalently the number of non-zero intervals), between the authocomplements of adjacent elements; the intuitive distance $\mathfrak{d}(\varphi a, \varphi c)$, that is, is generally unconstrained by the value of $\mathfrak{d}(a, c)$. In these circumstances the idea of adding a correction term to axiom (A1) seems distinctly unpromising.

The simplest policy, which is also the most conservative one, therefore seems to be to abandon the axiom system $\mathbb{A}$ altogether, to replace $(\mathbb{C} 0)$ by $\left(\mathbb{C} 0^{+}\right)$, and to add the isotony axiom $\left(\mathbb{C}^{\star} 4\right)$. It can then be shown, in a standard manner, that the new system, which we may call $\mathbb{C}+$, is logically equivalent to the system $\mathbb{B}$ : that $\mathbb{C}+$ implies $\mathbb{B}$ is the content of Birkhoff op.cit., Theorem X.1, while the proof that $\mathbb{B}$ implies $\mathbb{C}+$ is contained within lines $0-15$ and 35-40 of Miller (1984), § 2. (In these lines nothing more is assumed about $\mathfrak{L}$ than that it is a lattice. There is an error at lines 7f., which is implicitly corrected on p. 346 above.) But as announced in $\S 0$, our purpose here is to preserve the spirit of the system $\mathbb{A}$ by introducing a variant of axiom $(\mathbb{A} 2)$, and at the same time strengthening $(\mathbb{A} 1)$ to $\left(\mathbb{A}^{\star} 1\right)$. This variation will enable us to prove the equivalence of the new system $\mathbb{A}^{\star}$ with the system $\mathbb{C}^{\star}$, which consists of the same axioms as the old system $\mathbb{C}$, together with the isotony axiom $\left(\mathbb{C}^{\star} 4\right)$. What we have to surrender, in order to achieve this, is equivalence with the system $\mathbb{B}$, since its characteristic axiom $(\mathbb{B} 2)$ has to be so harshly mutilated that it no longer qualifies for participation in a respectable axiomatic system. But fortunately the variant $\mathbb{B}^{\natural}$ is at hand. If we modify its axiom $\left(\mathbb{B}^{\natural} 4\right)$, which is identical with axiom $(\mathbb{A} 2)$, in an identical way, we obtain a system $\mathbb{B}^{\text {t`}}$ completely equivalent with $\mathbb{A}^{\star}$ and $\mathbb{C}^{\star}$.

\section{Radial Convexity Refined}

The axioms of the new system $\mathbb{C}^{\star}$ are those of $\mathbb{C}$, augmented with an axiom of isotony $\left(\mathbb{C}^{\star} 4\right)$.

\begin{tabular}{|c|c|c|c|}
\hline$\left(\mathbb{C}^{\star} 0\right)$ & $\mathfrak{d}(a, c)$ & $={ }_{\mathrm{Df}}$ & $\mu(a \triangle c)$ \\
\hline$\left(\mathbb{C}^{\star} 1\right)$ & $\mu(\perp)$ & $=$ & 0 \\
\hline$\left(\mathbb{C}^{\star} 2\right)$ & $\mu(b)$ & $\geq$ & 0 \\
\hline$\left(\mathbb{C}^{\star} 3\right)$ & $\mu(a)+\mu(c)$ & $=$ & $\mu(a+c)+\mu(a \cdot c)$ \\
\hline$\left(\mathbb{C}^{\star} 4\right)$ & $a \succeq c$ & $\Rightarrow$ & $\mu(a) \geq \mu(c)$. \\
\hline
\end{tabular}

Principia 13(3): 339-56 (2009). 
The following derivations are conducted within this system $\mathbb{C}^{\star}$, together with some of the laws of Brouwerian algebra listed above. Applying $\left(\mathbb{C}^{\star} 3\right)$ and the definition $\left(\mathbb{C}^{\star} 0\right)$ to $(\mathbb{D} 7)$, we obtain

$\left(\mathbb{C}^{\star} 5\right) \quad a \succeq b \succeq c \Rightarrow \mu(a \Delta c)=\mu(a \Delta b)+\mu(b \Delta c)-\mu((a \Delta b) \cdot(b \Delta c))$,

$\left(\mathbb{C}^{\star} 6\right) \quad a \succeq b \succeq c \Rightarrow \mathfrak{d}(a, c)=\mathfrak{d}(a, b)+\mathfrak{d}(b, c)-\mu((a \Delta b) \cdot(b \Delta c))$.

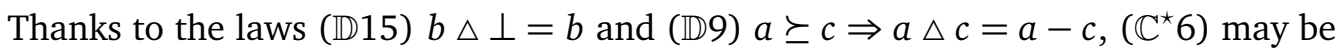
rewritten:

$\left(\mathbb{C}^{\star} 7\right) \quad a \succeq b \succeq c \Rightarrow \mathfrak{d}(a, c)=\mathfrak{d}(a, b)+\mathfrak{d}(b, c)-\mathfrak{d}((a \Delta b) \cdot(b \Delta c), \perp) ;$

$\left(\mathbb{C}^{\star} 8\right) \quad a \succeq b \succeq c \Rightarrow \mathfrak{d}(a, c)=\mathfrak{d}(a, b)+\mathfrak{d}(b, c)-\mathfrak{d}((a-b) \cdot(b-c), \perp)$.

Since $(a-b) \cdot(b-c)=\perp$ is not a Brouwerian theorem, even when $a \succeq b \succeq c$ (its dual is: if $p \vdash q \vdash r$ then $\vdash(r \rightarrow q) \vee(q \rightarrow p)$, which is intuitionistically invalid, though classically valid), the final term in $\left(\mathbb{C}^{\star} 8\right)$ will be greater than 0 for any metric $\mathfrak{d}$; that is, for any pseudometric that satisfies $(\mathbb{E} 9)$. In Brouwerian algebras no metric constrained by $\mathbb{C}^{\star}$ can be radially convex.

Our proposal - it may seem quite $a d$ hoc — is to modify the system $\mathbb{A}$ by replacing $(\mathbb{A} 2)$ with $\left(\mathbb{C}^{\star} 8\right)$, which is a Boolean equivalent. This new axiom, unlike $(\mathbb{A} 2)$, makes sense only in lattices in which the remainder operation is defined. The axioms of the revised system $\mathbb{A}^{\star}$ are:

$$
\begin{aligned}
& \mu(b)={ }_{\text {Df }} \mathfrak{d}(b, \perp) \\
& \left(\mathbb{A}^{\star} 1\right) \quad \mathfrak{d}(a, c)+\mathfrak{d}(\varphi a, \psi a) \geq \mathfrak{d}(\varphi c, \psi c) \\
& \left(\mathbb{A}^{\star} 2\right) \quad a \succeq b \succeq c \Rightarrow \mathfrak{d}(a, c)=\mathfrak{d}(a, b)+\mathfrak{d}(b, c)- \\
& -\mathfrak{d}((a-b) \cdot(b-c), \perp)
\end{aligned}
$$$$
\left(\mathbb{A}^{\star} 3\right) \quad \mathfrak{d}(b, b)=0 .
$$

Note that axiom $\left(\mathbb{A}^{\star} 3\right)$ must be stated independently. Without it, $\mathfrak{d}$ could be any constant non-zero function. (Thanks to $\left(\mathbb{A}^{\star} 2\right)$, and (D13), it suffices to postulate $\mathfrak{d}(\perp, \perp)=0$.) The abbreviation

$$
\kappa(a, b, c)=_{\mathrm{Df}} \mathfrak{d}((a-b) \cdot(b-c), \perp)
$$

allows $\left(\mathbb{A}^{\star} 2\right)$ to be expressed more concisely. The correction term $\kappa(a, b, c)$ may be thought of as a measure of the curvature (the deviation from linearity) of chains from $a$ to $c$ that pass through the point $b$. Since $(T-b) \cdot(b-\perp)=b^{\prime} \cdot b \succeq$ $(a-b) \cdot(b-c)$, the curvature $\kappa(a, b, c)$ is bounded above by $\mathfrak{d}\left(b \cdot b^{\prime}, \perp\right)$, which implies that $\kappa(a, b, c)=0$ if $b$ has a Boolean complement (that is, if $b \cdot b^{\prime}=\perp$ ). As explained 
in $\S 0$ above, the deductive theories of a language form a Brouwerian algebra, while according to Tarski (1935/1936), Theorem 17, the axiomatizable deductive theories are precisely those that conform to the law of non-contradiction. We may therefore combine the languages of general metamathematics and general relativity to say (not entirely seriously) that Boolean elements (propositions) possess zero mass, and only unaxiomatizable theories can engender deflections from straightness. Note, however, that the converse fails: $(a-b) \cdot(b-c)=\perp$ does not imply $b \cdot b^{\prime}=\perp$. For example, if $a=b$ or $b=c$, then by $(\mathbb{D} 13)$ and $\left(\mathbb{A}^{\star} 0\right), \kappa(a, b, c)=0$.

In Boolean algebras the systems $\mathbb{A}^{\star}$ and $\mathbb{C}^{\star}$ reduce to $\mathbb{A}$ and $\mathbb{C}$ respectively. We turn now to the task of proving that in Brouwerian algebras $\mathbb{A}^{\star}$ and $\mathbb{C}^{\star}$ are equivalent to each other, starting with the proof that $\mathbb{C}^{\star}$ implies $\mathbb{A}^{\star}$. It follows at once from $(\mathbb{D} 15)$ and $\left(\mathbb{C}^{\star} 0\right)$ that $\left(\mathbb{A}^{\star} 0\right)$ holds, and from $(\mathbb{D} 16),\left(\mathbb{C}^{\star} 0\right)$, and $\left(\mathbb{C}^{\star} 1\right)$ that $\left(\mathbb{A}^{\star} 3\right)$ holds. $\left(\mathbb{A}^{\star} 2\right)$ is $\left(\mathbb{C}^{\star} 8\right)$. It remains to prove $\left(\mathbb{A}^{\star} 1\right)$.

We have seen that $\left(\mathbb{A}^{\star} 0\right)$ follows from $\left(\mathbb{C}^{\star} 0\right)$ and $(\mathbb{D} 15)$, and from the three of them together,

$$
\mathfrak{d}(a, c)=\mathfrak{d}(a \triangle c, \perp) .
$$

By appealing to $(\mathbb{D} 7),\left(\mathbb{C}^{\star} 4\right)$, and $\left(\mathbb{A}^{\star} 0\right)$ we establish easily the second isotony principle (E24),

$$
a \succeq b \succeq c \Rightarrow \mathfrak{d}(a, c) \geq \mathfrak{d}(b, c),
$$

and by appealing to $\left(\mathbb{C}^{\star} 0\right),\left(\mathbb{C}^{\star} 3\right),\left(\mathbb{C}^{\star} 2\right),(\mathbb{D} 6)$, and $\left(\mathbb{C}^{\star} 4\right)$, we establish triangularity as easily,

$\left(\mathbb{C}^{\star} 11\right) \mathfrak{d}(b, a)+\mathfrak{d}(b, c)=\mu(a \triangle b)+\mu(b \triangle c)$

$$
\geq \mu((a \Delta b)+(b \Delta c) \geq \mu(a \Delta c)=\mathfrak{d}(a, c) .
$$

Writing for $\chi$ in $(\mathbb{D} 10)$ the term $\varphi \Delta \psi$, and then applying $\left(\mathbb{C}^{\star} 10\right)$, ( $\left.\mathbb{C}^{\star} 9\right)$, and $\left(\mathbb{C}^{\star} 11\right)$, we obtain

$$
\begin{aligned}
\mathfrak{d}(a \Delta c, \perp) & \geq \mathfrak{d}((\varphi a \Delta \psi a) \Delta(\varphi c \Delta \psi c), \perp), \\
\mathfrak{d}(a \Delta c, \perp) & \geq \mathfrak{d}(\varphi a \Delta \psi a, \varphi c \Delta \psi c), \\
\mathfrak{d}(a \Delta c, \perp)+\mathfrak{d}(\varphi a \Delta \psi a, \perp) & \geq \mathfrak{d}(\varphi c \Delta \psi c, \perp) .
\end{aligned}
$$

A further three-fold application of $\left(\mathbb{C}^{\star} 9\right)$ establishes our old principle of partial diversity $\left(\mathbb{A}^{\star} 1\right)$ :

$$
\mathfrak{d}(a, c)+\mathfrak{d}(\varphi a, \psi a) \geq \mathfrak{d}(\varphi c, \psi c)
$$

We turn now to the converse derivation, the derivation of the axioms of $\mathbb{C}^{\star}$ within the system $\mathbb{A}^{\star}$. Since $\left(\mathbb{A}^{\star} 3\right)$ is the same as $(\mathbb{E} 6)$, and $\left(\mathbb{A}^{\star} 1\right)$ is the same as $(\mathbb{E} 7)$, the Principia 13(3): 339-56 (2009). 
metrical formulas $(\mathbb{E} 6)-(\mathbb{E} 24)$ of $\S 2$ can all be called on for this purpose. Axiom $\left(\mathbb{C}^{\star} 1\right)$ is an immediate consequence of $\left(\mathbb{A}^{\star} 0\right)$ and $\left(\mathbb{A}^{\star} 3\right)$. Formula $(\mathbb{E} 15)$ establishes that $\mathfrak{d}$ is non-negative, and by $\left(\mathbb{A}^{\star} 0\right)$ the same holds for $\mu$, establishing $\left(\mathbb{C}^{\star} 2\right)$. It follows from $(\mathbb{E} 24)$ that if $a \succeq c$ then $\mathfrak{d}(a, \perp) \geq \mathfrak{d}(c, \perp)$, and hence by $\left(\mathbb{A}^{\star} 0\right)$ that $\mu(a) \geq \mu(c)$; that is to say, $\mu$ is isotone, as stated in $\left(\mathbb{C}^{\star} 4\right)$. For $\left(\mathbb{C}^{\star} 0\right)$ and $\left(\mathbb{C}^{\star} 3\right)$ rather more work is needed.

To establish $\left(\mathbb{C}^{\star} 0\right)$, we first prove within the system $\mathbb{A}^{\star}$ that $\mathfrak{d}(a+c, a \cdot c)=$ $\mathfrak{d}(a \Delta c, \perp)$. By the Lipschitz condition $(\mathbb{E} 14), \mathfrak{d}(a \Delta c, \perp) \geq \mathfrak{d}((a \Delta c)+c, \perp+c)$,

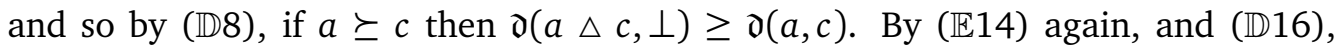
$\mathfrak{d}(a, c) \geq \mathfrak{d}(a \Delta c, c \Delta c)=\mathfrak{d}(a \Delta c, \perp)$. Since $a+c \succeq a \cdot c$, these last two results establish that $\mathfrak{d}((a+c) \Delta a \cdot c, \perp)=\mathfrak{d}(a+c, a \cdot c)$. By $(\mathbb{D} 4)$, we conclude that $\mathfrak{d}(a \triangle c, \perp)=\mathfrak{d}(a+c, a \cdot c)$. It was shown in formula (A8) on p. 344 that the equality of the diagonals of the lattice quadrangles is derivable from $\left(\mathbb{A}^{\star} 1\right)$ alone: $\mathfrak{d}(a, c)=$ $\mathfrak{d}(a+c, a \cdot c)$. It follows that $\mathfrak{d}(a, c)=\mathfrak{d}(a \Delta c, \perp)$ and, by application of $\left(\mathbb{A}^{\star} 0\right)$, the proof of $\left(\mathbb{C}^{\star} 0\right)$ is complete.

In any Brouwerian algebra, by $(\mathbb{D} 1)$ and $(\mathbb{D} 14),((a+c)-c) \cdot(c-\perp)=(a-c) \cdot c$. By $(\mathbb{D} 2),(\mathbb{D} 14)$, and $(\mathbb{D} 3),(a-a \cdot c) \cdot(a \cdot c-\perp)=(a-c) \cdot(a \cdot c)=(a-c) \cdot c$. In other words, by $\left(\mathbb{A}^{\star} 4\right), \kappa(a+c, c, \perp)=\kappa(a, a \cdot c, \perp)$. The chain $a+c \rightsquigarrow c \rightsquigarrow \perp$ has the same curvature at $c$ as the chain $c \rightsquigarrow a \cdot c \rightsquigarrow \perp$ has at $a \cdot c$, and if we apply $\left(\mathbb{A}^{\star} 2\right)$ to each chain, and subtract, the curvature terms cancel out:

$\left(\mathbb{A}^{\star} 5\right) \mathfrak{d}(a+c, \perp)-\mathfrak{d}(a, \perp)=\mathfrak{d}(a+c, c)+\mathfrak{d}(c, \perp)-\mathfrak{d}(a, a \cdot c)-\mathfrak{d}(a \cdot c, \perp)$.

By $(\mathbb{E} 22)$, we may infer that $\mathfrak{d}(a+c, \perp)-\mathfrak{d}(a, \perp)=\mathfrak{d}(c, \perp)-\mathfrak{d}(a \cdot c, \perp)$. The definition $\left(\mathbb{A}^{\star} 0\right)$ may now be applied to each term, with the conclusion $\left(\mathbb{C}^{\star} 3\right): \mu(a)+\mu(c)=$ $\mu(a+c)+\mu(a \cdot c)$.

The modified axiomatic systems $\mathbb{A}^{\star}$ and $\mathbb{C}^{\star}$ are thus interderivable. They are interderivable also with the system $\mathbb{B}^{\natural \star}$, which is obtained from $\mathbb{B}^{\natural}$ by modifying $\left(\mathbb{B}^{\natural} 4\right)$ in the same way as $(\mathbb{A} 2)$ was modified $\left(\left(\mathbb{B}^{\natural \star} 2\right)\right.$ is indeed the same formula as $\left(\mathbb{A}^{\star} 2\right)$ ). The proofs of $\mathbb{B}^{\natural \star}$ from $\mathbb{A}^{\star}$ and of $\mathbb{C}^{\star}$ from $\mathbb{B}^{\text {}}$ are minor variations on proofs that have been given earlier. The details are omitted.

\section{Metrization}

A measure $\mu$ for which the system $\left(\mathbb{C}^{\star}\right)$ holds is called strictly positive whenever $\mu(b)=0$ implies that $b=\perp$. That is, by $\left(\mathbb{C}^{\star} 0\right)$, the pseudometric $\mathfrak{d}$ is a metric that obeys ( $\mathbb{E} 9$ ). Conversely, if $\mathfrak{d}$ is a metric, the measure $\mu$ defined by $\left(\mathbb{A}^{\star} 0\right)$ is positive isotone. The Brouwerian algebra $\mathfrak{L}$ can be metrized in accordance with system $\mathbb{A}^{\star}$ if and only if it admits a metric that satisfies the axioms of $\mathbb{A}^{\star}$; that is, if and only if it admits a strictly positive measure $\mu$.

Principia 13(3): 339-56 (2009). 
The algebras that concern us, the algebras of theories of a classical logical calculus based on a denumerably infinite language, are all atomic, the atoms being the maximal theories. These algebras either contain a theory that cannot be finitely maximized (that is, extended to a maximal theory by adjoining a single proposition), or contain no such theory. According to a theorem of Mostowski (1937), the algebras in the first class, which are epitomized by any calculus vulnerable to Gödel's theorem, have $2^{\aleph_{0}}$ atoms, while those in the second class have $\aleph_{0}$ atoms. For details of Mostowski's results, and a relatively elementary proof, see Miller (1992).

Theorem 2.5 of Horn \& Tarski (1948) states that an algebra $\mathfrak{T}$ of theories that has denumerably many atoms admits a strictly positive measure, and is thus metrizable in accordance with the system $\mathbb{A}^{\star}$. But a theory algebra with $2^{\aleph_{0}}$ atoms admits no such measure, and is not metrizable in accordance with the system $\mathbb{A}^{\star}$.

It does not follow that there are algebras of classical theories that are not metrizable. In general, metrization demands less than measurability, as the following intuitive remarks may help to make clear. Because distinct theories imply distinct propositions, of which there are only denumerably all told, every path from $\top$ to $\perp$ in an algebra of theories has at most $\aleph_{0}$ links. A radially convex metric therefore must divide the interval $[\top, \perp]$ into at most $\aleph_{0}$ disjoint pieces. But an algebra with continuum many atoms is pear-shaped, very much wider at the bottom than it is tall, and the total available measure on it must be divided into non-denumerably many non-zero pieces.

Making use of results of Urysohn, Carruth, and Vietoris, Mormann (2006), Theorem 5.4, has demonstrated that each algebra of theories $\mathfrak{T}$ is metrizable by a radially convex metric $\partial$ (a metric satisfying $\mathbb{A} 2$ ). We shall use Mormann's theorem to show that each algebra $\mathfrak{T}$ of theories is metrizable in another way by a metric $\mathfrak{d}$ that satisfies $\left(\mathbb{A}^{\star} 1\right)$ and for which, for every $a, c \in \mathfrak{T}$,

$$
\mathfrak{d}(a, c)=\partial(a \triangle c, \perp) .
$$

Since $\mathfrak{T}$ may not be measurable, we cannot use the definition $\left(\mathbb{A}^{\star} 0\right)$ to define a measure $\mu$ in terms of $\mathfrak{d}$ (or in terms of $\partial$ either). It follows that $\left(\mathbb{A}^{\star} 2\right.$ ) must fail for $\mathfrak{d}$. The system $\mathbb{A}^{\star}$ was designed specifically to be interderivable with $\mathbb{C}^{\star}$, but the design must now be partly unstitched.

The correction term $\kappa$ in $\left(\mathbb{A}^{\star} 2\right)$ was calculated (p.351) by applying $\left(\mathbb{C}^{\star} 3\right)$ and $\left(\mathbb{C}^{\star} 0\right)$ to $(\mathbb{D} 7)$. If we are given a bounded function $v$, not a measure, nothing need stop us from recalculating the correction term to suit a different purpose. Let $\lambda(a, c)$ record the extent to which the function $v$ deviates from additivity, so that

$$
v(a)+v(c)=v(a+c)+v(a c)+\lambda(a, c) .
$$

We can preserve the axiom scheme $\left(\mathbb{A}^{\star} 1\right)$ if we replace $\left(\mathbb{A}^{\star} 2\right)$ by

$\left(\mathbb{A}^{\lambda} 2\right) a \succeq b \succeq c \Rightarrow \mathfrak{d}(a, c)=\mathfrak{d}(a, b)+\mathfrak{d}(b, c)-\kappa(a, b, c)-\lambda(a-b, b-c)$.

Principia 13(3): 339-56 (2009). 
If the function $\lambda$ can be expressed in terms of $\mathfrak{d}$, then of course it should be so expressed. It follows that the theory $\mathbb{C}^{\lambda}$, whose axioms are $\left(\mathbb{C}^{\star} 0\right),\left(\mathbb{C}^{\star} 1\right),\left(\mathbb{C}^{\star} 2\right)$, $\left(\mathbb{C}^{\lambda} 3\right)$, implies the theory $\mathbb{A}^{\lambda}$, whose axioms are $\left(\mathbb{A}^{\star} 0\right)\left(\mathbb{A}^{\star} 1\right),\left(\mathbb{A}^{\lambda} 2\right)$, and $\left(\mathbb{A}^{\star} 3\right)$. The converse implication does not hold.

Mormann's proof is non-constructive, and in view of the extraordinary variety of theory algebras with $2^{\aleph_{0}}$ atoms, it is unlikely that much can be said in general about the metric $\partial$. My interest here is only in the values $v(b)=\partial(b, \perp)$, which are all positive and bounded by $\partial(T, \perp)$. Thanks to $\left(\mathbb{C}^{\star} 0\right)$ and $\left(\mathbb{A}^{\star} 6\right)$, we can define in terms of this function $v$ a metric $\mathfrak{d}$ that, in almost the same way as before, may be proved to satisfy the theory $\mathbb{A}^{\lambda}$, and in particular axiom $\left(\mathbb{A}^{\star} 1\right)$.

THEOREM: Let $\mathfrak{T}$ be a Brouwerian algebra of theories. If $\partial$ is a metric on $\mathfrak{T}$, then there exists a metric $\mathfrak{d}$ on $\mathfrak{T}$ that satisfies axiom $\left(\mathbb{A}^{\star} 1\right)$ and for which $\mathfrak{d}(a, c)=\partial(a \triangle c, \perp)$ for each $a, c \in \mathfrak{T}^{1}$

\section{References}

Birkhoff, G. 1967. Lattice Theory. 3rd edition. Providence RI: American Mathematical Society. Horn, A. \& Tarski, A. 1948. Measures in Boolean Algebras. Transactions of the American Mathematical Society 64: 467-97.

Miller, D. W. 1977. On Distance from the Truth as a True Distance. (short version) Bulletin of the Section of Logic, Institute of Philosophy \& Sociology, Polish Academy of Sciences, Wrocław 6(1): 15-26.

-1978. On Distance from the Truth as a True Distance. In K. J. J. Hintikka, I. M. O. Niiniluoto, \& E. Saarinen (eds.) Essays on Mathematical \& Philosophical Logic, pp. 415435. Dordrecht: D. Reidel Publishing Company.

- 1979. Metric Postulates for Modular, Distributive, and Boolean Lattices. Bulletin of the Section of Logic, Institute of Philosophy \& Sociology, Polish Academy of Sciences, Wrocław 8(4): 191-6.

- 1984. A Geometry of Logic. In H. J. Skala, S. Termini, \& E. Trillas (eds.) Aspects of Vagueness, pp. 91-104. Dordrecht: D. Reidel Publishing Company.

- 1986. Continuous Connectives. Fisal-84 [Fall International Seminar on Applied Logic] Proceedings, pp. 173-179. Palma de Mallorca: Govern Balear Conselleria d'Educació i Cultura, Universitat de les Illes Balears.

- 1992. The Disposition of Complete Theories. Coleção Documentos, Série Lógica e Teoria da Ciência, 10, agosto de 1992, Instituto de Estudos Avançados, Universidade de São Paulo. Available at http://www.warwick.ac.uk/go/dwmiller/usp.pdf/.

- 1994. Critical Rationalism. A Restatement and Defence. Chicago \& La Salle: Open Court Publishing Company.

Mormann T. 2006. Truthlikeness for Theories on Countable Languages. In I. C. Jarvie, K. M. Milford, \& D. W. Miller (eds.) Karl Popper: A Centenary Assessment. Volume III: Science, pp. 3-15. Aldershot \& Burlington VT: Ashgate. 
Mostowski, A. 1937. Abzählbare Boolesche Körper und ihre Anwendung auf die allgemeine Metamathematik. Fundamenta Mathematicae 29(1): 34-53. English translation 1979. Foundational Studies. Selected Works, Volume II, pp.1-17. Amsterdam: North-Holland Publishing Company, and Warsaw: Polish Scientific Publishers.

Nordhaus, E. A. \& Lapidus, L. 1954. Brouwerian Geometry. Canadian Journal of Mathematics 6: 217-229.

Tarski, A. 1935/1936. Grundzüge des Systemenkalkül. Fundamenta Mathematicae 25(4): 503-26, and 26(2): 283-301. English translation 1956. Logic, Semantics, Metamathematics, pp. 342-383. Oxford: Clarendon Press. Second edition 1983. Indianapolis: Hackett Publishing Company.

\author{
DAVID MiLler \\ Department of Philosophy \\ University of Warwick \\ Coventry CV4 7AL UK \\ dwmiller57@yahoo.com
}

Resumo. A fim de medir o grau de dessemelhança entre elementos de uma álgebra booleana, o autor propôs em (1984) usar pseudométricas satisfazendo generalizações dos axiomas usuais para a identidade. A proposta é estendida, na medida em que é exequível, de álgebras booleanas (álgebras de proposições) para álgebras de Brouwer (álgebras de teorias dedutivas). A relação entre geometrias booleanas e de Brouwer da lógica resulta semelhante, de maneira curiosa, à relação entre geometrias euclidianas e não-euclidianas do espaço físico. $\mathrm{O}$ artigo conclui com uma breve consideração do problema da metrização da álgebra de teorias.

Palavras-chave: Álgebra booleana, álgebra de Brouwer, métrica, mensuração, identidade parcial.

\title{
Notes
}

${ }^{1}$ Some of this material has been presented at the International Conference on Algebraic \& Topological Methods in Non-Classical Logics at Tbilisi State University, in 2003, and at the XIV Encontro Brasileiro De LóGiCA at Itatiaia in 2006; and also at seminars at the Department of Mathematics, University of Warwick, in 2005, at the Departamento de Filosofía, Universidad Nacional de Córdoba, Argentina, and the Departamento de Matemáticas, Universidad Nacional de Colombia, Bogotá, in 2006. It is a real pleasure to be able to dedicate this written version to Newton da Costa on his 80th birthday. In preparing it, I have been much encouraged by the interest shown by Thomas Mormann in the geometry of logic. As always, responsibility for errors is reserved.

Principia 13(3): 339-56 (2009). 